Published in final edited form as:

Bone. 2007 August ; 41(2): 282-289.

\title{
Effects of long-term estrogen replacement therapy on the prevalence and area of periarticular tibial osteophytes in surgically postmenopausal cynomolgus monkeys
}

\author{
Erik J. Olson ${ }^{1}$, Bruce R. Lindgren ${ }^{2}$, and Cathy S. Carlson ${ }^{1}$ \\ 1Department of Veterinary Population Medicine University of Minnesota, St. Paul, MN \\ 2School of Public Health University of Minnesota, Minneapolis, MN
}

\section{Abstract}

Osteoarthritis $(\mathrm{OA})$ occurs naturally in cynomolgus macaques. The purposes of the present study were to: 1) develop histological measurement schemes to measure the cross-sectional area of axial and abaxial osteophytes in the proximal tibia; 2) determine the effects of long-term estrogen replacement therapy (ERT) on osteophyte prevalence and area; and 3) assess relationships between osteophyte size and risk factors of OA (age and body weight) and concurrent bone and cartilage lesions. Adult female cynomolgus macaques $(\mathrm{n}=180)$ were bilaterally ovariectomized $(\mathrm{OVX})$ and were treated for three years with ERT, soy phytoestrogens (SPE), or no hormones (OVX controls). At necropsy, the prevalence and cross-sectional area of periarticular tibial osteophytes were evaluated histologically. Treatment effects on osteophyte prevalence and area were evaluated using Chi-square analyses and Kruskal-Wallis test, respectively; other comparisons were evaluated using regression analyses. The prevalence of abaxial osteophytes in the medial tibial plateau was not significantly affected by treatment group; however, the prevalence of abaxial osteophytes in the lateral tibial plateau was significantly lower in ERT group than SPE group ( $p<0.01)$. The total number of abaxial osteophytes (sum of lateral and medial) was significantly lower in ERT group compared to OVX and SPE groups. Neither the prevalence of axial osteophytes nor the sum of lateral and medial axial osteophytes was significantly affected by treatment in either tibial plateau. There were no significant treatment effects on axial or abaxial osteophyte cross-sectional area in either tibial plateau. There were significant positive correlations between abaxial osteophyte area and SCB thickness in the medial tibial plateau ( $\mathrm{p}=0.048$ ); however, there were no significant correlations between abaxial osteophyte area (medial or lateral) and age or body weight. In this model of naturally occurring OA, long-term ERT does not consistently reduce the prevalence, and has no significant effects on cross-sectional area, of periarticular tibial osteophytes.

\section{Keywords}

animal models; bone histomorphometry; estrogen; osteoarthritis; primate

\footnotetext{
Corresponding Author: Erik J. Olson, DVM Department of Veterinary Population Medicine College of Veterinary Medicine University of Minnesota 244 Veterinary Diagnostic Laboratory 1333 Gortner Avenue St. Paul, MN 55108 Tel: 612-624-1709 Fax: 612-624-8707 E-mail: olso1183@umn.edu

Erik J. Olson: olso1183@umn.edu Bruce R. Lindgren: lindg001@umn.edu Cathy S. Carlson: carls099@umn.edu

Conflict of Interest: All authors have no conflict of interest.

Publisher's Disclaimer: This is a PDF file of an unedited manuscript that has been accepted for publication. As a service to our customers we are providing this early version of the manuscript. The manuscript will undergo copyediting, typesetting, and review of the resulting proof before it is published in its final citable form. Please note that during the production process errors may be discovered which could affect the content, and all legal disclaimers that apply to the journal pertain.
} 


\section{Introduction}

Periarticular osteophytes are frequently associated with osteoarthritis (OA) and appear as outgrowths of cartilage at the margins of joints which subsequently undergo endochondral ossification [1,2]. Despite being one of the primary clinical/radiographic criteria in defining $\mathrm{OA}$, the role of periarticular osteophytes in the pathophysiology and progression of OA is currently unknown. It remains unclear whether osteophytes are a concomitant event of OA (epiphenomenon) or if they are part of a reactive process in an unstable joint.

The teleological purpose of osteophytes is unclear, although it is thought that periarticular osteophytes may stabilize an osteoarthritic joint [2]. For example, joint movement, such as that occurring with a ruptured anterior (cranial) cruciate ligament in humans and in natural and experimental animal (canine and rodent) models of OA, tends to promote osteophyte formation in the knee (stifle) joint [2-7]. It has been shown that these osteophytes limit the translocation of the femur on the tibia and stabilize the joint in the sagittal plane [2]. Conversely, experimental models of OA in dogs suggest that immobilization of the knee joint inhibits osteophyte development [8].

The relationship between periarticular osteophytes and damage to or loss of articular cartilage also is unclear. Some authors have suggested that osteophyte formation is part of the remodeling process following loss of articular cartilage [5,9]. In a mouse model, van Osch et al.[10] showed a good correlation between osteophyte size and cartilage damage; however, others have suggested that osteophytes may develop without the loss of articular cartilage [11].

Recent evidence suggests that osteophytes are strongly associated with malalignment to the side of the osteophyte (ipsilateral side) and it is thought that this is related to an increase in force over the given side of the joint [2]. For example, abaxial (marginal) osteophytes in the medial compartment of the human knee (distal femur and proximal tibia) tended to be associated with varus malalignment. The authors hypothesized that osteophytes may not have a primary effect themselves, but rather that they serve as markers for two factors that strongly affect the risk of progression, namely limb malalignment and pre-existing cartilage loss [2].

Factors other than biomechanical forces also have been proposed in the development and pathogenesis of periarticular osteophytes. For example, recent literature suggests that macrophages (from periosteum or synovium) may play a crucial role in osteophyte development through their production of growth factors such as TGF- $\beta$ [12-17]. Evidence that TGF- $\beta$ plays a role in osteophyte development includes the finding that multiple intra-articular injections of TGF- $\beta$ or adenoviral overexpression of TGF- $\beta 1$ in the knee joint resulted in the formation of osteophytes in rats and mice [14,15]. In addition, data from experimental models of OA in rats suggest that bone resorption may be involved in the local activation and release of TGF- $\beta$, promoting osteophyte formation $[6,7,15,18-20]$. Inhibition of endogenous TGF- $\beta$ using soluble receptor (TGF-B-RII) nearly completely prevented osteophyte formation in an experimental model of OA in rats; however, this treatment also resulted in a reduction in the thickness of articular cartilage [13]. Hormones such as parathyroid hormone-related protein (PTHrP) and growth factors such as fibroblast growth factor receptor 3 (FGFR3) and insulinlike growth factor I (IGF-I) have also been implicated in the development of osteophytes [21]. To our knowledge, the effects of estrogen on periarticular osteophytes in naturally occurring $\mathrm{OA}$, as were examined in the present study, have not been previously evaluated.

Obesity is a major modifiable risk factor of OA, and it has been shown that excessive weight increases one's risk of both incident OA and the progression of disease [22-28]. Interestingly, in an experimental model of OA (surgical transection of the cranial cruciate ligament) as well as in naturally occurring OA (spontaneous rupture of the cranial cruciate ligament) in dogs, 
the degree of osteophyte formation was more extensive in larger and more obese dogs [3,29]. These findings suggest that body weight alone or in combination with other factors contributes to the pathogenesis of OA and the development of periarticular osteophytes. Age is another important risk factor of OA;[26,30] however, age as a factor in the etiology of periarticular osteophytes has rarely been specifically examined. Some authors consider osteophytes in the knee to be primarily a phenomenon of aging;[11] however, to determine if this is true, the effects of age on the development and size of periarticular osteophytes would need to be studied independently of OA.

Several schemes and descriptive atlases have been published to evaluate osteophyte size using imaging modalities of the knee (radiographs and MRI); however, very few publications include detailed schemes for identifying and measuring periarticular osteophytes histologically. Atlases that include drawings of periarticular osteophytes are designed for radiographic interpretation, and the evaluation of osteophyte size typically involves a limited grading scale (e.g., 0-3; absent to severe) [31-33]. Previous histological studies of osteophytes do not include the details of the measurement schemes used to assess osteophyte size (area); specifically, the criteria that were used to distinguish the margins of surrounding native bone from those of osteophyte bone, $[7,34]$ which are necessary to obtain reproducible data from the osteophyte area measurements.

Naturally-occurring OA in cynomolgus monkeys has been utilized previously as a model of the human disease to evaluate the effects of estrogen replacement therapy (ERT) on the severity of articular cartilage lesions of OA [35]. Importantly, the prevalence and severity of OA in these animals increases with increasing age [36]. Older adult monkeys with severe radiographic OA have also been observed to have musculoskeletal clinical signs (e.g., limping, stiff gait) [35].

In the present study, osteophyte prevalence and size (cross-sectional area) were evaluated in the proximal tibia of surgically postmenopausal cynomolgus monkeys from a large, long-term study that was designed to evaluate the effects of ERT on coronary artery disease. A previous study focusing on OA lesions in these same tibiae demonstrated that articular cartilage lesions of OA were significantly reduced by treatment with ERT [35]. That study also reported that the prevalence of periarticular osteophytes was decreased in the ERT-treated animals; however, osteophyte location (axial vs. abaxial) was not recorded, only data from the medial tibial plateau were reported, and osteophyte cross-sectional area was not evaluated [35].

The purposes of the present study were to: 1) develop histological measurement schemes to measure the area of axial and abaxial osteophytes in lateral and medial proximal tibial plateaus; 2) determine the effects of ERT on osteophyte prevalence and size (area); 3) assess relationships between osteophyte size and risk factors of OA (age and body weight); and 4) assess relationships between osteophyte size and concurrent bone and cartilage lesions present in OA (e.g., articular cartilage fibrillation, chondrocyte clones, subchondral bone (SCB) thickness, calcified cartilage thickness). To assist the readers, the anatomic locations lateral and medial as well as SCB have been bolded and the terms axial and abaxial have been underlined throughout the manuscript.

\section{Materials and Methods}

\section{Animals}

The animals used in this study were from an experiment that was designed to evaluate the effects of estrogen deficiency, exogenous ERT, and soy phytoestrogen treatment (SPE) on atherosclerosis of the coronary arteries [37]. The original study included 180 feral adult female cynomolgus macaques and has previously been described in detail $[35,37]$. 


\section{Study design}

The animals were bilaterally ovariectomized to simulate menopause and were randomly divided into three age- and weight-matched treatment groups for a 36-month treatment period, during which all animals were fed a moderately atherogenic diet (40\% of calories from fat) containing $120 \mathrm{kcal} / \mathrm{kg}$ of body weight/day (approximately equivalent to $1800 \mathrm{kcal} / \mathrm{day}$ for the average woman in the United States) [37]. Group 1 (OVX control, $\mathrm{n}=60$ ) received no treatment, Group 2 (SPE, $\mathrm{n}=60$ ) received soy phytoestrogens (at $129 \mathrm{mg} /$ day human equivalent), and Group 3 (ERT, $n=60$ ) received conjugated equine estrogens [Premarin; Wyeth-Ayerst Laboratories, Philadelphia, PA] (at $0.625 \mathrm{mg} /$ day human equivalent) in the diet. Plasma hormone levels were measured during the 36-month treatment period to document effective treatment levels [37]. All animals were administered intravenous calcein $(10 \mathrm{mg} / \mathrm{kg}) 21$ and 7 days prior to necropsy. At the termination of the study, the mean age of the animals was 12.0 years (range: 9.6-15.8 years; standard deviation: 1.1), as estimated by dentition,[38] and their mean body weight was $3.3 \mathrm{~kg}$ (range: $2.1-6.2 \mathrm{~kg}$; standard deviation: 0.7 ).

\section{Necropsy and tissue preparation}

At necropsy, the right knee joints were collected and disarticulated. After removal of soft tissues, each proximal tibia was serially sectioned at 2-mm intervals using a diamond saw, and a midcoronal section from each set of serial sections was identified for histological evaluations and histomorphometry measurements. This tissue section/slab was decalcified, embedded in paraffin, sectioned at $6 \mu \mathrm{m}$, and stained with toluidine blue. Two slides per animal were evaluated, a toluidine blue-stained mid-coronal section and an H\&E-stained serial section. All sections were randomized and the evaluator (EO) was blinded to the treatment group assignments.

\section{Histological evaluation}

Proximal tibial sections from 165 of the original 180 monkeys (54 untreated controls, OVX; 57 receiving SPE; and 54 receiving ERT) were available for evaluation of the prevalence and size (cross-sectional area) of abaxial osteophytes in this study. For technical reasons (e.g., critical tissue missing from the block or plane of section issues), sections from a slightly lower number of animals (158 total, including 52 untreated controls, OVX; 53 receiving SPE; and 53 receiving ERT) were available for evaluation of axial osteophytes.

The prevalence of periarticular osteophytes (within $500 \mu \mathrm{m}$ of the articular surface) in the abaxial (peripheral/marginal) and axial (central; referred to as the relative prominence of central tibial spines in the human literature) aspects of the medial and lateral tibial plateaus was recorded. Abaxial osteophytes were defined as outgrowths of bone and cartilage at the joint margins of the proximal tibia. Both the overall shape and profile of the joint margin and the presence of areas of woven bone were used to evaluate the sections. Some abaxial osteophyte margins were histologically obvious due to well-demarcated shape changes of the joint that were comprised entirely of woven bone; however, other abaxial osteophytes were less obvious histologically due to smaller size and/or some degree of bone remodeling. There were similar numbers of histologically subtle abaxial osteophytes among the three treatment groups in both the medial and lateral tibial plateau. These osteophytes were identified based on comparisons to control tibiae (without periarticular osteophytes) and by estimating the overall change in shape of the joint profile. Because the normal shape of the axial aspect of the tibial plateau is not definitively known in this species (e.g., variable height of tibial spines), two separate evaluation schemes were used to evaluate the prevalence of the axial osteophytes: 1) only histologically obvious axial osteophytes (i.e. those with sharply demarcated margins that also had a vertical height (amplitude) greater than the total osteophyte width) and 2) any vertical deviation (in the y-axis/plane) from a flat tibial plateau. In locations in which an osteophyte was present, the total osteophyte cross-sectional area (including bone, marrow, and 
void spaces, $\mathrm{mm}^{2}$ ) was measured using a $2 \mathrm{X}$ objective with the Spot ${ }^{\circledR}$ Advanced morphometry software (Diagnostic Instruments, Sterling Heights, MI) and the following measurement schemes: 1) Abaxial osteophytes (away from the central axis of the tibia; marginal): The outer margin (i.e., most peripheral margin of calcified cartilage) of each abaxial osteophyte was hand-traced with a computer mouse starting at the transition from articular (hyaline) cartilage to fibrocartilage and ending at disto-lateral margin of the osteophyte. A straight line was then drawn from this point back to the tracing start point and the total enclosed area was calculated by the morphometry software (Figs. 1,2), and 2) Axial osteophytes (adjacent to the central axis of the tibia): A vertical line was drawn through the midline of the proximal tibia, starting at the calcified cartilage-bone interface (base of calcified cartilage) and ending at the upper margin of the calcified cartilage. Next, the outer margin of the osteophyte was hand-traced following the upper margin of the calcified cartilage. Because the axial osteophytes were usually more poorly defined (i.e., less obvious), two separate measurement methods were used to determine the landmarks for the lower margin/measurement (i.e., the base) of the osteophytes: Method A) a more conservative method which only included histologically obvious axial osteophytes, and Method B) a more liberal measurement that included any deviation from a flat articular surface.

Axial Method A-This measurement scheme took into account any histologically obvious axial osteophyte. In tracing along the deep margin (interface with SCB) of the osteophyte, the measurement line was drawn straight across at points where bony trabeculae were narrower than their overall height (Figs. 3,4).

Axial Method B-Using the more liberal method $(\operatorname{Method} B)$, the termination of the measurement was taken at the level directly across (lateral or medial) from the starting point at the calcified cartilage-bone junction. A line was then drawn connecting the termination measurement with the starting point, following the inner/distal subchondral bone outline (Figs. $5,6)$. Figure 5 represents an osteophyte in which the termination of the measurement was relatively obvious; however, other axial osteophytes were much more subtle, and the points of termination were not as obvious.

The total number ( 0 or 1 per plateau) of abaxial osteophytes present in each tibial plateau (medial and lateral) was summed to derive the total number of abaxial (0-2) and axial (0-2) osteophytes in each section of proximal tibia. In addition, the individual abaxial and axial osteophyte areas from each tibial plateau were added together to derive the total abaxial and axial osteophyte areas for each section of proximal tibia.

Finally, the SCB thickness in the lateral tibial plateau was evaluated using Osteomeasure histomorphometry software (Osteometrics, Atlanta, GA) with a $2 \mathrm{X}$ objective as described previously [35]. Briefly, the margins were hand-traced with a computer mouse in a $3.5 \times 3.5$ $\mathrm{mm}$ field in the center of the tibial plateau (included approximately $80 \%$ of the SCB of the tibial plateau).

\section{Statistical analyses}

The Chi-square test was used to compare treatment effects on the prevalence of axial and abaxial osteophytes, either individually (within the lateral or medial plateau) or as a sum of the lateral and medial tibial plateaus. Area and thickness measurements, along with other quantitative variables were evaluated for treatment effects using analysis of variance (ANOVA) and regression techniques. If any of the measurements had a non-normal distribution (highly skewed to high or low values), a data transformation, such as the logarithmic transformation, was performed. If an appropriate transformation was not found, non-parametric methods such as the Kruskal-Wallis one-way ANOVA and the Spearman 
correlation were applied. Adjustments were made for post-hoc testing using either the Bonferroni or the Tukey's studentized range procedures for multiple comparisons, which maintain the overall level of significance at 0.05 . All statistical analyses were performed using SAS version 9.1 (SAS Institute Inc., Cary, NC).

The OA histological grading and measurement data that were previously described by Ham et al. were re-analyzed in the subset of sections containing an abaxial osteophyte in the medial tibial plateau using principal components (factor) analysis [35]. The medial tibial plateau was chosen because, as in previous studies, the OA lesions were more severe in this site than in the lateral tibial plateau [35]. Abaxial osteophytes were chosen for evaluation because the abaxial osteophytes in these sections were much more well-defined than the axial osteophytes. This resulted in a reduction in the number of specimens available for evaluation (compared with the study by Ham et al.),[35] since only 108 of the original 166 sections contained medial abaxial osteophytes. The variables that were included in the factor analysis included the following: OA severity (including articular cartilage structure score; loss of toluidine blue staining; number of chondrocyte clones; maximum number of tidemarks; number of blood vessels crossing the tidemark; number of chondrified blood vessels); $\mathbf{S C B}$ area, thickness, and a subjective evaluation of SCB thickness (SCB score); calcified cartilage area and thickness; and articular cartilage area and thickness. Using an orthogonal rotation method (varimax), these initial linear combinations of the variables were rotated to create independent factors. The subset of variables that had a factor loading greater than the absolute value of 0.3 was used to represent each factor. Regression analysis was done to evaluate the potential relationships between medial abaxial osteophyte area and the factors.

\section{Results \\ Osteophyte prevalence}

The sections examined contained 108 medial abaxial osteophytes and 133 lateral abaxial osteophytes (Table 1). The prevalence of abaxial osteophytes in the medial tibial plateau was not significantly affected by treatment group; however, the prevalence of abaxial osteophytes in the lateral tibial plateau was significantly lower in the ERT group than in the SPE group $(p<0.01)$ (Table 1). Although not statistically significant, there was a trend toward a lower prevalence of lateral abaxial osteophytes in the ERT group than in the OVX control group. The total number of abaxial osteophytes (sum of lateral and medial abaxial osteophytes) also was significantly affected by treatment, being lower in the ERT group than both the OVX control and SPE treatment groups ( $\mathrm{p}<0.01$ for both; data not shown). The prevalence of lateral axial osteophytes using the conservative (axial method A) and liberal (axial method B) methods were exactly the same (no additional axial osteophytes identified with the liberal method); however, there was a marked difference between the prevalence of medial axial osteophytes identified using the two measurement schemes, with 32, 30, and 38 additional medial axial osteophytes identified in each of the three treatment groups (OVX, SPE, ERT, respectively) using the more liberal measurement scheme (axial method B) compared to the conservative scheme (axial method A). The prevalence of axial osteophytes was not significantly affected by treatment in the medial or lateral tibial plateau using either the conservative (axial method A) or liberal (axial method B) measurement schemes (Table 1) nor were there significant treatment differences in the total number of axial osteophytes (sum of lateral and medial axial osteophytes) using either measurement scheme.

\section{Osteophyte area}

Lateral abaxial osteophytes were noticeably larger than the medial abaxial osteophytes (Table 1). Among the 133 specimens with abaxial osteophytes (out of the total 165 specimens examined), lateral abaxial osteophyte area ranged from 0.06 to $3.51 \mathrm{~mm}^{2}$ (mean $=0.77$ 
$\mathrm{mm}^{2}$ ). Among the 108 specimens with medial abaxial osteophytes (out of the total 165 specimens examined), medial abaxial osteophyte area ranged from 0.02 to $1.65 \mathrm{~mm}^{2}$ (mean $=0.43 \mathrm{~mm}^{2}$ ). There were no significant treatment effects on abaxial osteophyte area in any location (lateral or medial; Table 1) nor were there significant treatment effects on the total abaxial osteophyte area (sum of lateral and medial plateaus; data not shown). In contrast to the abaxial osteophytes, lateral axial osteophytes were considerably smaller (approximately one-half the size) than the medial axial osteophytes using either measurement scheme. Among the 158 specimens examined, lateral axial osteophyte area (using the conservative axial method A) ranged from 0.10 to $1.94 \mathrm{~mm}^{2}\left(\right.$ mean $\left.=0.59 \mathrm{~mm}^{2}\right)$ and the area of the medial axial osteophytes ranged from $0.22 \mathrm{~mm}^{2}$ to $2.16 \mathrm{~mm}^{2}$ (mean $=1.10 \mathrm{~mm}^{2}$ ). Lateral axial osteophyte area (using the liberal axial method B) ranged from 0.10 to $1.94 \mathrm{~mm}^{2}$ (mean $=0.59$ $\mathrm{mm}^{2}$ ) and medial axial osteophyte area (also using the liberal axial method B) ranged from $0.22 \mathrm{~mm}^{2}$ to $3.21 \mathrm{~mm}^{2}$ (mean $=1.15 \mathrm{~mm}^{2}$ ). There were no significant treatment effects on axial osteophyte area (lateral and medial) using either of the axial measurement schemes (Table 1) nor were there significant treatment effects on total axial osteophyte area (sum of lateral and medial tibial plateaus; data not shown) using either of the measurement schemes (methods A and B).

As described previously,[39] there was a significant weight gain among the monkeys during the three-year study; however, there were no significant differences in initial or terminal body weights among the three treatment groups (data not shown). There was no significant correlation between either lateral or medial abaxial osteophyte area and body weight or age at the termination of the study. As previously reported, the SCB thickness in the medial tibial plateau of the original data set $(n=166)$ was lowest in the OVX control group and highest in the ERT group,[35] and this also was true in the lateral tibial plateau, but the differences were not significant. In addition, there were no statistically significant treatment group differences in either lateral or medial SCB thickness in the subset of sections with either lateral $(n=133)$ or medial $(\mathrm{n}=108)$ abaxial osteophytes. The mean thickness of the $\mathbf{S C B}$ was nearly $50 \%$ greater in the medial tibial plateau compared to that of the lateral tibial plateau in all three treatment groups $(207.7 \mu \mathrm{m}$ in the lateral tibial plateau and $376.2 \mu \mathrm{m}$ in the medial tibial plateau across all three treatment groups). In the subset of sections with medial abaxial osteophytes ( $\mathrm{n}=108)$, there was a significant positive correlation between the medial SCB thickness and medial abaxial osteophyte area ( $\mathrm{p}=0.048)$. In the subset of sections with lateral abaxial osteophytes $(n=133)$; however, there was no significant correlation between the SCB thickness of the lateral tibial plateau and lateral abaxial osteophyte area.

\section{Description of factors}

Principal components analysis using the data from sections of medial tibial plateaus containing abaxial osteophytes $(n=108)$ identified four factors that explained $74.6 \%$ of the variability in the data (Table 2). Factor 1 was weighted by the SCB parameters (SCB area, SCB thickness, SCB score) and this factor accounted for $21.3 \%$ of the variance in the data (Table 2). Factor 2 was weighted primarily by articular cartilage thickness and area, and accounted for $19.4 \%$ of the variance in the data. The differences in OA severity (weighted primarily by scores for articular cartilage structure, number of chondrocyte clones, and loss of toluidine blue staining) were explained by factor 3 (17.4\% of variance). Factor 4 was weighted by calcified cartilage thickness and area and explained $16.5 \%$ of the variance in the data.

There was a significant positive correlation $(\mathrm{p}<0.05)$ between medial abaxial osteophyte area and factor 2 (weighted by articular cartilage thickness and area) and also between medial abaxial osteophyte area and factor 3 (weighted by OA severity scores). There were no significant correlations between medial abaxial osteophyte area and factor 1 (weighted by SCB thickness and area) or factor 4 (weighted by calcified cartilage thickness and area). 
Treatment group was not significantly associated $(\mathrm{p}>0.1)$ with medial abaxial osteophyte area after controlling for each of the four factors in separate regression models.

\section{Discussion}

This study utilized well-defined, objective and reproducible measurement schemes to identify periarticular osteophytes and to evaluate their cross-sectional area in both the axial and abaxial aspects of the proximal tibial plateau. Abaxial osteophytes in the proximal tibial plateau were relatively common in these monkey tibiae, being present in approximately $80 \%$ of the sections in the lateral tibial plateau and in approximately $65 \%$ of the medial tibial plateau sections. With one exception (significantly fewer lateral abaxial osteophytes in ERT group compared with SPE group), the prevalence of abaxial osteophytes in either the medial or lateral tibial plateau was not affected by treatment group. Only when the total number of abaxial osteophytes in each tibia (sum of abaxial osteophytes in lateral and medial tibial plateau) was evaluated was there a significant reduction in the ERT group compared with both the OVX and SPE groups. The lack of consistency in these results suggests that estrogen deficiency does not strongly influence the prevalence of abaxial osteophytes in either the medial or lateral tibial plateaus. In support of this argument, the prevalence of axial osteophytes was not significantly affected by treatment, regardless of location (medial or lateral) or measurement scheme (methods A and B). The reason that there were differences in the prevalence of axial osteophytes using the two measurement schemes (100 additional axial osteophytes were identified using the more liberal method B) may be related to the overall shape of the axial osteophytes in the lateral versus the medial tibial plateau in monkeys. The more liberal method B included any deviation from a flat, horizontal line as an axial osteophyte and it is possible that the lateral tibial plateau tends to be less flat than the medial tibial plateau in this species.

Had the animals been older at the time the study was initiated, pre-existing osteophytes would have been quite likely and an effect of hormonal treatment on osteophyte prevalence, in the absence of baseline data, would have been more difficult to assess. However, given the relatively young age of the monkeys (the mean age at study initiation was 9.0 years which is equivalent to approximately 27 human years), it is unlikely that many of these animals had pre-existing osteophytes [11]. The 3-year duration of the study also increased the chances of observing a treatment effect on osteophyte prevalence. Since no consistent treatment effect was detected, it appears that estrogen deficiency does not have a strong influence on the development of periarticular osteophytes.

The fact that there were no significant treatment effects on abaxial or axial osteophyte area in either the medial or lateral tibial plateau suggests that, even though ERT leads to decreased severity of articular cartilage lesions of OA in cynomolgus monkeys, factors other than ERT are more important in the growth (cross-sectional area) of periarticular osteophytes in ovariectomized cynomolgus macaques. In humans, limb alignment and biomechanical forces have been implicated in determining the area of periarticular osteophytes in the knee. Although there was a trend for larger medial abaxial osteophytes to be present in monkeys with increasing terminal body weight, the results were not statistically significant. In addition, there was no significant correlation between lateral abaxial osteophyte area and body weight. In addition, age did not correlate with either medial or lateral abaxial osteophyte size/area. It is important to note, however, that the monkeys in the present study had relatively narrow ranges for both age and body weight. None of the monkeys were obese, and nearly all of the monkeys were middle-aged, making this data set less than ideal for evaluating these associations.

As was reported for the medial tibial plateau previously,[35] the lateral SCB was thinnest in the OVX group and thickest in the ERT group. There also was a significant positive correlation 
between medial abaxial osteophyte area and medial SCB thickness $(\mathrm{p}<0.05)$; however, there was no significant correlation between lateral abaxial osteophyte area and lateral SCB thickness. This discrepancy may be explained by the fact that OA lesions, in both monkeys and humans, occur first and tend to be more severe in the medial tibial plateau.

There was a significant positive correlation between factor 3 (OA severity) and medial abaxial osteophyte area, i.e., larger medial abaxial osteophytes were associated with sites having more severe lesions of OA. This is consistent with the fact that the most severe OA lesions occur in the medial tibial plateau. The correlation between factor 3 (OA severity) and medial abaxial osteophyte cross-sectional area may also suggest that biomechanical factors influence both articular cartilage lesions and osteophyte size.

The positive correlation between factor 2 (articular cartilage thickness) and medial abaxial osteophyte area is not as easily explained. Inspection of the data did not reveal a clear association between articular cartilage thickness and osteophyte size. Although these findings appear to be contradictory, a possible explanation is that the articular cartilage lesions of OA were mild in the majority of these cases and the relatively thick articular cartilage likely represents swelling. The correlation analysis also includes all of the variables that comprise each factor and in fact, when examined using correlation analysis, medial abaxial osteophyte area was not significantly correlated with either articular cartilage width $(\mathrm{p}=0.227)$ or articular cartilage area $(\mathrm{p}=0.056)$ when these parameters were evaluated individually.

Factor 1 (weighted primarily by $\mathbf{S C B}$ thickness) was not significantly correlated with medial abaxial osteophyte area; however, medial SCB thickness alone was significantly positively correlated with medial abaxial osteophyte area, although the correlation was relatively weak $(\mathrm{r}=0.19)$. It is likely that the other variables in factor 1 somewhat 'diluted' the correlation with medial abaxial osteophyte area due to the complex interactions of each variable. Therefore, the two comparisons are different in that one evaluates the correlation with a composite variable and the other examines a very specific bivariate relationship. These results suggest that SCB thickness is not strongly associated with osteophyte size and that factors influencing SCB thickness likely are different from those determining osteophyte size.

Measurement of osteophyte area in histological sections allows for a high degree of accuracy in two dimensions, but does not allow evaluation of osteophyte volume. Evaluation of multiple sections over a larger area of the proximal tibia would have provided a more accurate evaluation of osteophyte size in three dimensions and may be considered in future studies. In order to standardize the assessments that were done in the present study, however, two serial sections of one midcoronal slab from each site were evaluated. Another limitation of the study was that baseline data on the osteophytes were not available. This type of analysis would require highdetail imaging modalities that were not readily available at the time the study was initiated. Finally, the methods used to measure osteophyte area evaluated the contribution of bone, marrow space, and void space together without distinguishing these individual anatomic components.

In spite of these limitations, these data represent a unique sample of tibial sections from a relatively large-scale, long-term controlled study using weight-, age-, and diet-matched nonhuman primates. Detailed methods (including identification criteria and measurement schemes), which could easily be modified for evaluation of osteophytes in other anatomic sites, were devised to evaluate and measure periarticular tibial osteophytes.

The overall findings of this experiment suggest that neither long-term ERT nor estrogen deficiency strongly influence the prevalence or area of periarticular proximal tibial osteophytes. Combined with these results, the lack of a consistent effect of age or weight on 
osteophyte size suggests that biomechanical instability may be an important determinant of osteophyte size, as has been hypothesized previously in humans.

\section{Acknowledgments}

The authors thank Josh Parker and Drs. Kim Ham and Laura Eikmeier for technical assistance. We also thank Dr. Thomas Clarkson for providing the knee joints for this study, Dr. Mary Anthony for providing the details of the clinical trial, and Jean Gardin for assistance with tissue collection. This study was supported by National Institutes of Health Grants RR14099 and RR18719.

\section{References}

1. Sharma L, Song J, Felson DT, Cahue S, Shamiyeh E, Dunlop DD. The role of knee alignment in disease progression and functional decline in knee osteoarthritis. JAMA 2001;286:188-195. [PubMed: 11448282]

2. Felson DT, Gale DR, Elon Gale M, Niu J, Hunter DJ, Goggins J, LaValley MP. Osteophytes and progression of knee osteoarthritis. Rheumatology 2005;44:100-104. [PubMed: 15381791]

3. Heffron LE, Campbell JR. Osteophyte formation in the canine stifle joint following treatment for rupture of the cranial cruciate ligament. J Small Anim Pract 1979;20:603-611. [PubMed: 513655]

4. Marshall JL. Periarticular osteophytes: Initiation and formation in the knee of the dog. Clin Orthop Relat Res 1969;62:37-47. [PubMed: 4975461]

5. Gilbertson EMM. Development of periarticular osteophytes in experimentally induced osteoarthritis in the dog. Ann Rheum Dis 1975;34:12-25. [PubMed: 1124952]

6. Hayami T, Pickarski M, Wesolowski GA, Mclane J, Bone A, Destefano J, Rodan GA, Duong LT. The role of subchondral bone remodeling in osteoarthritis. Arthritis Rheum 2004;50:1193-1206. [PubMed: 15077302]

7. Hayami T, Pickarski M, Zhou Y, Wesolowski GA, Rodan GA, Duong LT. Characterization of articular cartilage and subchondral bone changes in the rat anterior cruciate ligament transection and meniscectomized models of osteoarthritis. Bone 2006;38:234-243. [PubMed: 16185945]

8. Palmoski MJ, Brandt KD. Immobilization of the knee prevents osteoarthritis after anterior cruciate ligament transection. Arthritis Rheum 1982;25:1201-1208. [PubMed: 7138593]

9. Moskowitz RW. Bone remodeling in osteoarthritis: Subchondral and osteophytic responses. Osteoarthritis Cartilage 1999;7:323-324. [PubMed: 10329315]

10. van Osch GJ, van der Kraan PM, van Valburg AA, van den Berg WB. The relation between cartilage damage and osteophyte size in a murine model for osteoarthritis in the knee. Rheumatol Int 1996;16:115-119. [PubMed: 8893376]

11. Hernborg J, Nilsson BE. The relationship between osteophytes in the knee joint, osteoarthritis and aging. Acta Orthop Scand 1973;44:69-74. [PubMed: 4702611]

12. Klocke NW, Snyder PW, Widmer WR, Zhong W, McCabe GP, Breur GJ. Detection of synovial macrophages in the joint capsule of dogs with naturally occurring rupture of the cranial cruciate ligament. AJVR 2005;66:493-499.

13. Scharstuhl A, Glansbeek HL, van Beuningen HM, Vitters EL, van der Kraan PM, van den Berg WB. Inhibition of endogenous TGF- $\beta$ during experimental osteoarthritis prevents osteophyte formation and impairs cartilage repair. J Immunol 2002;169:507-514. [PubMed: 12077282]

14. van Beuningen HM, Glansbeek HL, van der Kraan PM, van den Berg WB. Osteoarthritis like changes in the murine knee joint resulting from intra-articular transforming growth factor-beta injections. Osteoarthritis Cartilage 2000;8:25-33. [PubMed: 10607496]

15. Bakker AC, van de Loo FA, van Beuningen HM, Sime P, van Lent PL, van der Kraan PM, Richards $\mathrm{CD}$, van den Berg WB. Overexpression of active TGF-beta-1 in the murine knee joint: Evidence for synovial-layer-dependent chondro-osteophyte formation. Osteoarthritis Cartilage 2001;9:128-136. [PubMed: 11237660]

16. Blom AB, van Lent PL, Holthuysen AE, van der Kraan PM, Roth J, van Rooijen N, van den Berg WB. Synovial lining macrophages mediate osteophyte formation during experimental osteoarthritis. Osteoarthritis Cartilage 2004;12:627-635. [PubMed: 15262242] 
17. van Lent PL, Blom AB, van der Kraan P, Holthuysen AE, Vitters E, van Rooijen N, Smeets RL, Nabbe KC, van den Berg WB. Crucial role of synovial lining macrophages in the promotion of transforming growth factor beta-mediated osteophyte formation. Arthritis Rheum 2004;50:103-111. [PubMed: 14730606]

18. Cameron ML, Fu FH, Paessler HH, Schneider M, Evans CH. Synovial fluid cytokine concentrations as possible prognostic indicators in the ALC-deficient knee. Knee Surg Sports Traumatol Arthrosc 1994;2:38-44. [PubMed: 7584175]

19. Manicourt DH, Altman RD, Williams JM, Devogelaer JP, Druetz-Van Egeren A, Lenz ME, Pietryla D, Thonar EJ. Treatment with calcitonin suppresses the responses of bone, cartilage, and synovium in the early stages of canine experimental osteoarthritis and significantly reduces the severity of the cartilage lesions. Arthritis Rheum 1999;42:1159-1167. [PubMed: 10366108]

20. Schlaak JF, Pfers I. Meyer Zum Buschenfelde KH, Marker-Hermann E. Different cytokine profiles in the synovial fluid of patients with osteoarthritis, rheumatoid arthritis and seronegative spondylarthropathies. Clin Exp Rheumatol 1996;14:155-162. [PubMed: 8737721]

21. Huch K, Kleffner S, Stöve J, Puhl W, Günther K-P, Brenner RE. PTHrP, PTHr, and FGFR3 are involved in the process of endochondral ossification in human osteophytes. Histochem Cell Biol 2003;119:281-287. [PubMed: 12692671]

22. Manninen P, Riihimaki H, Heliovarra M, Makela P. Overweight, gender, and knee osteoarthritis. Int J Obes Relat Metabol Disord 1996;20:595-597.

23. Felson DT, Anderson JJ, Naimark A, Walker AM, Meenan RF. Obesity and knee osteoarthritis. The Framingham study. Ann Intern Med 1988;109:18-24. [PubMed: 3377350]

24. Felson DT, Zhang Y, Anthony JM, Naimark A, Anderson JJ. Weight loss reduces the risk for symptomatic knee osteoarthritis in women. The Framingham Study. Ann Intern Med 1992;116:535539. [PubMed: 1543306]

25. Felson DT, Zhang Y, Hannan MT, Naimark A, Weissman B, Aliabadi P, Levy D. Risk factors for incident radiographic knee osteoarthritis in the elderly: The Framingham study. Arthritis Rheum 1997;40:728-733. [PubMed: 9125257]

26. Felson DT, Lawrence RC, Dieppe PA, Hirsch R, Helmick CG, Jordan JM, Kington RS, Lane NE, Nevitt MC, Zhang Y, Sowers M, McAlindon T, Spector TD, Poole AR, Yanovski SZ, Ateshian G, Sharma L, Buckwalter JA, Brandt KD, Fries JF. Osteoarthritis: New insights, part 1: The disease and its risk factors. Ann Intern Med 2000;133:635-646. [PubMed: 11033593]

27. Dougados M, Gueguen A, Nguyen M, Thiesce A, Listrat V, Jacob L, Nakache JP, Gabriel KR, Lequesne M, Amor B. Longitudinal radiologic evaluation of osteoarthritis of the knee. J Rheumatol 1992;19:378-383. [PubMed: 1578451]

28. Schouten JS, van den Ouweland F, Valkenburg HA. A 12 year follow up study in the general population on prognostic factors of cartilage loss in osteoarthritis of the knee. Ann Rheum Dis 1992;51:932-937. [PubMed: 1417116]

29. Paatsama S, Sittnikow K. Early changes in the knee joint due to instability induced by cutting of the anterior cruciate ligament: An experimental study in young dogs. Acta Radiol Supp 1972;319:169173.

30. Felson DT, Zhang Y, Hannan MT, Naimark A, Weissman BN, Aliabadi P, Levy D. The incidence and natural history of knee osteoarthritis in the elderly. Arthritis Rheum 1995;38:1500-1505. [PubMed: 7575700]

31. Altman RD, Hochberg M, Murphy WA, Wolfe F, Lequesne M. Atlas of individual radiographic features in osteoarthritis. Osteoarthritis Cartilage 1995;3(Suppl A):3-70. [PubMed: 8581752]

32. Nagaosa Y, Mateus M, Hassan B, Lanyon P, Doherty M. Development of a logically devised line drawing atlas for grading of knee osteoarthritis. Ann Rheum Dis 2000;59:587-595. [PubMed: 10913052]

33. Nagaosa Y, Lanyon P, Doherty M. Characterisation of size and direction of osteophyte in knee osteoarthritis: A radiographic study. Ann Rheum Dis 2002;61:319-324. [PubMed: 11874834]

34. Ono M, Yamashita Y, Minamizaki T, Morio Y. Processes of osteophyte formation in guinea pigs with spontaneous osteoarthritis. Yonago Acta Medica 2000;43:131-140. 
35. Ham KD, Loeser RF, Lindgren BR, Carlson CS. Effects of long-term estrogen replacement therapy on osteoarthritis severity in cynomolgus monkeys. Arthritis Rheum 2002;46:1956-1964. [PubMed: 12124881]

36. Carlson CS, Loeser RF, Purser CB, Gardin JF, Jerome CP. Osteoarthritis in cynomolgus macaques. III. Effects of age, gender, and subchondral bone thickness on severity of disease. J Bone Miner Res 1996;11:1209-1217. [PubMed: 8864894]

37. Clarkson TB, Anthony MS, Moran TM. Inhibition of postmenopausal atherosclerosis progression: A comparison of the effects of conjugated equine estrogens and soy phytoestrogens. J Clin Endocrinol Metab 2001;86:41-47. [PubMed: 11231976]

38. Bowen WH, Koch G. Determination of age in monkeys (Macaca irus) on the basis of dental development. Lab Anim 1970;4:113-124. [PubMed: 5005918]

39. Ham KD, Carlson CS. Effects of estrogen replacement therapy on bone turnover in subchondral bone and epiphyseal metaphyseal cancellous bone of ovariectomized cynomolgus monkeys. J Bone Miner Res 2004;19:823-829. [PubMed: 15068506] 


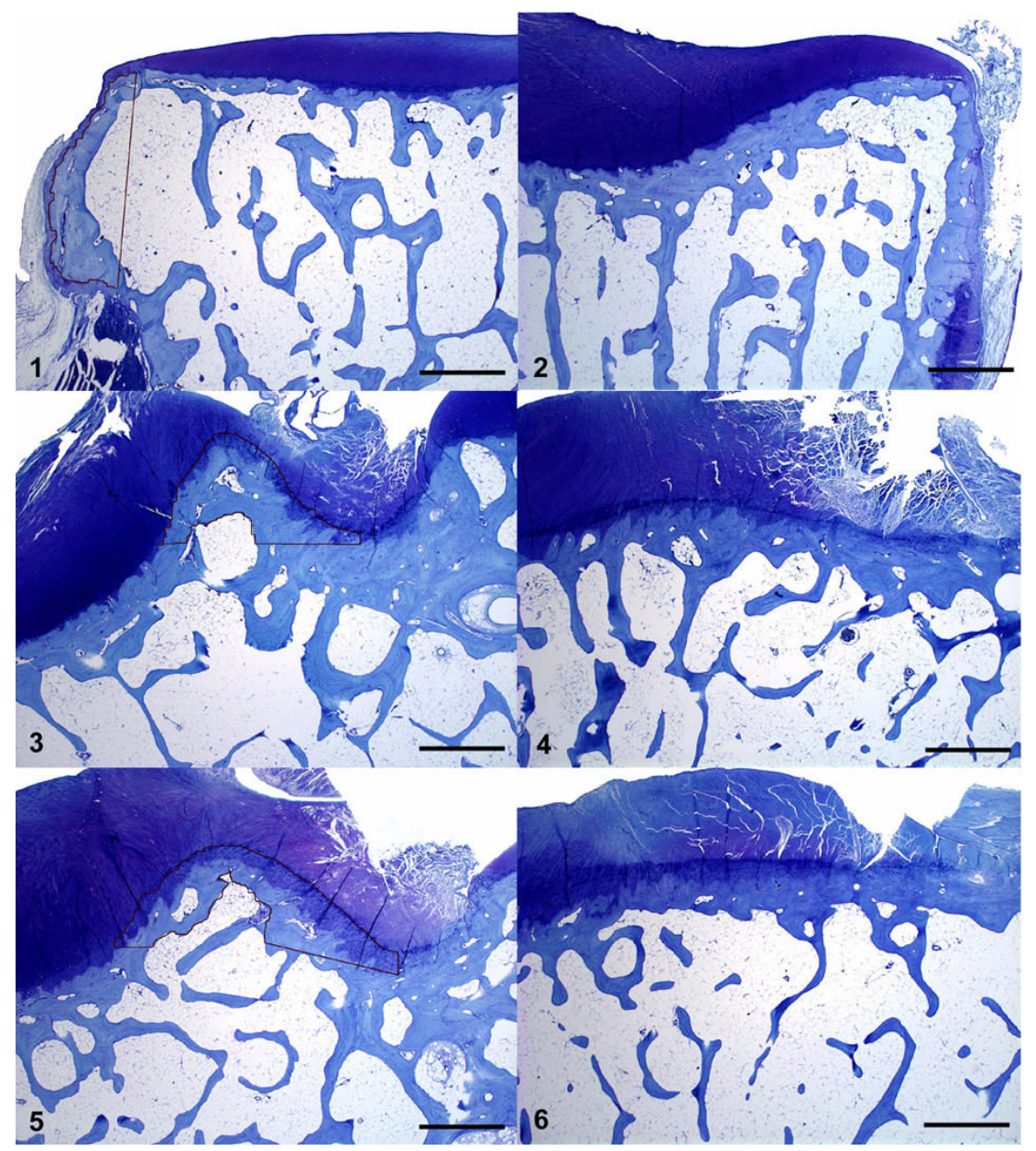

Figures 1-6. Cynomolgus monkey, proximal tibiae, toluidine blue stain, $2 \mathrm{X}$ objective

Fig. 1. Lateral abaxial compartment. Black tracing outlines margin of well-defined, lateral abaxial osteophyte. Toluidine blue. Bar $=1000 \mu \mathrm{m}$. Fig. 2. Medial abaxial compartment. Note absence of obvious abaxial osteophyte; hyaline cartilage extends to abaxial margin of specimen. Toluidine blue. Bar $=1000 \mu \mathrm{m}$. Fig. 3. Medial axial compartment. Black tracing outlines well-defined medial axial osteophyte using measurement method A. Toluidine blue. Bar $=1000 \mu \mathrm{m}$. Fig. 4. Medial axial compartment. Note absence of well-defined medial axial osteophyte; the surface is relatively flat. Toluidine blue. Bar $=1000 \mu \mathrm{m}$. Fig. 5. Medial axial compartment. Black tracing outlines a more subtle medial axial osteophyte using measurement method B. Toluidine blue. Bar $=1000 \mu \mathrm{m}$. Fig. 6. Medial axial compartment. Note absence of well-defined medial axial osteophyte; the surface is relatively flat. Toluidine blue. Bar $=1000$ $\mu \mathrm{m}$. 


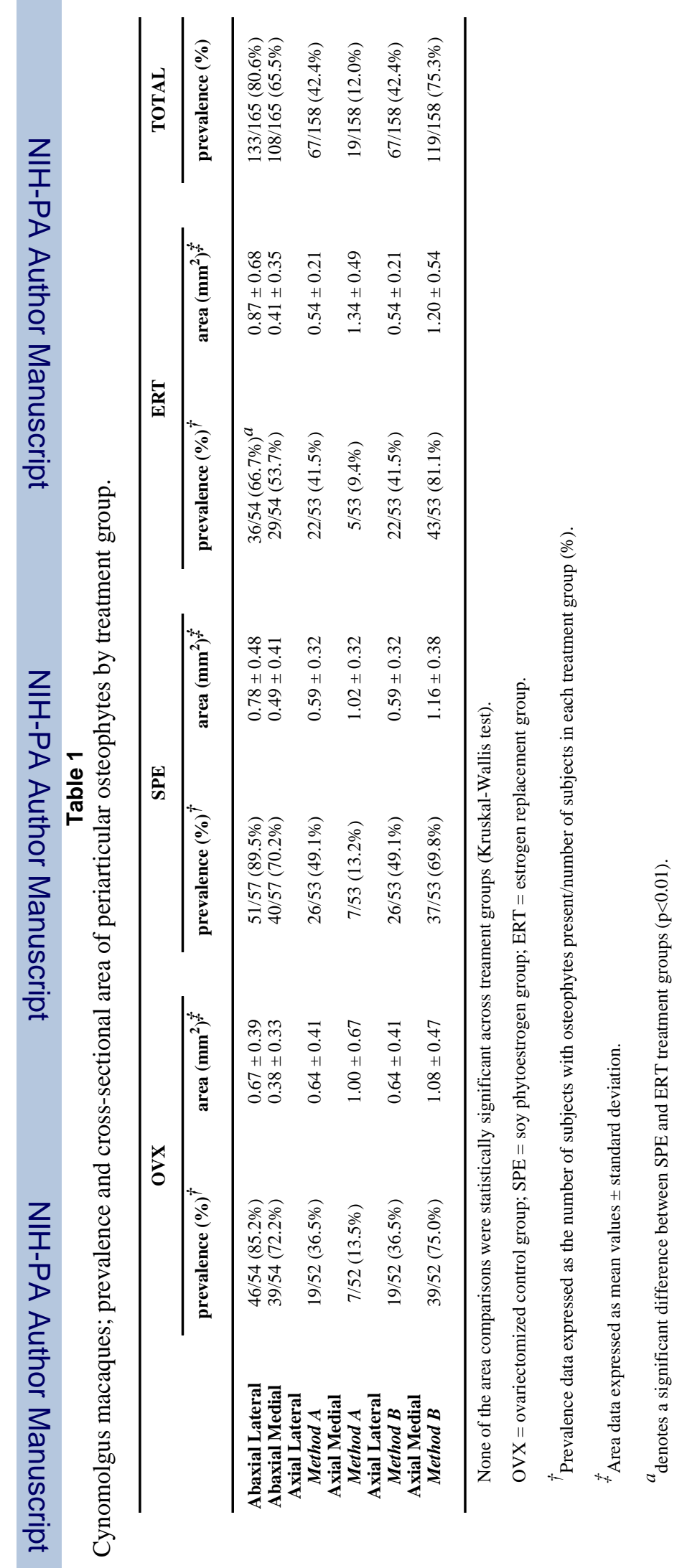

Bone. Author manuscript; available in PMC 2008 August 1. 
Table 2

Results of the factor analysis for individual histomorphometry parameters; monkey knees (medial tibial plateau).

\begin{tabular}{|c|c|c|c|}
\hline Factor & $\begin{array}{c}\% \text { of variance } \\
\text { explained }\end{array}$ & Factor composition & Factor loading $^{\dagger}$ \\
\hline \multirow[t]{2}{*}{$\begin{array}{l}\text { Factor } 1 \text { (SCB } \\
\text { thickness) }\end{array}$} & 21.3 & $\mathrm{SCB}$ area, $\mathrm{mm}^{2}$ & $\overline{0.96}$ \\
\hline & & $\begin{array}{l}\text { SCB width/thickness, } \mu \mathrm{m} \\
\text { SCB score }\end{array}$ & $\begin{array}{l}0.95 \\
0.89\end{array}$ \\
\hline \multirow[t]{3}{*}{ Factor 2 (AC thickness) } & 19.4 & $\mathrm{AC}$ area, $\mathrm{mm}^{2}$ & 0.88 \\
\hline & & $\begin{array}{c}\text { AC width/thickness, } \mu \mathrm{m} \\
\text { chondrified blood vessels, no. }\end{array}$ & $\begin{array}{l}0.83 \\
0.69\end{array}$ \\
\hline & & blood vessels crossing tidemark, no. & 0.61 \\
\hline \multirow[t]{5}{*}{ Factor 3 (OA severity) } & 17.4 & AC structure, score & 0.80 \\
\hline & & $\begin{array}{l}\text { loss of toluidine blue staining, score } \\
\text { chondrocyte clones, no. }\end{array}$ & $\begin{array}{l}0.75 \\
0.75\end{array}$ \\
\hline & & blood vessels crossing tidemark, no. & 0.41 \\
\hline & & AC width/thickness, $\mu \mathrm{m}$ & -0.37 \\
\hline & & chondrified blood vessels & 0.31 \\
\hline \multirow[t]{3}{*}{ Factor 4 (CC thickness) } & 16.5 & $\mathrm{CC}$ width/thickness, $\mu \mathrm{m}$ & 0.96 \\
\hline & & $\mathrm{CC}$ area, $\mathrm{mm}^{2}$ & 0.96 \\
\hline & & tidemarks, no. & 0.39 \\
\hline
\end{tabular}

$\mathrm{AC}=$ articular cartilage; $\mathrm{CC}=$ calcified cartilage; $\mathrm{OA}=$ osteoarthritis; $\mathrm{SCB}=$ subchondral bone.

${ }^{\dagger}$ Only the factor loadings greater than the absolute value of 0.30 are shown. 